\title{
Editors Note
}

With this issue, ALBION is changed. One new feature which we hope from time to time can be repeated is the special issue devoted to a particular subject. This issue is entitled "Violence and Social Control." In five articles beginning with that by George Rudé we find further evidence of the turbulent evolution of British society. We are confident that this special issue is an important contribution to the ongoing study of violence and social control in western societies. We believe also, that this completely revised format will be appreciated by our readers, and that it will support the quality of ALBION's contents.

$$
\begin{aligned}
& \text { B.M.G. } \\
& \text { J.M.R. } \\
& \text { M.J.M. }
\end{aligned}
$$

